\title{
Terapia de reperfusión en infarto agudo al miocardio. Comparación entre trombolisis endovenosa y angioplastía coronaria
}

\author{
HÉCTOR UGALDE, DIEGO UGALDE ${ }^{a}$, MACARENA MUÑOZ ${ }^{a}$
}

\section{Angioplasty compared to thrombolysis as the initial reperfusion therapy in acute myocardial infarction}

Background: Primary angioplasty is superior to intravenous thrombolysis as reperfusion therapy for acute myocardial infarction. Aim: To compare the results of available reperfusion strategies for initial management of acute myocardial infarction during hospitalization and 5 years follow up. Patients and Methods: Historical cohort study from a prospective registry of patients admitted with acute myocardial infarction to our center. Patients treated with primary angioplasty were identified and were then matched by age, sex and date of event with patients treated with thrombolysis. The clinical outcomes were compared including hospitalization and 5-years follow-up. Results: From March 1993 to August 2001, 98 patients were treated with primary angioplasty and matched with 98 thrombolyzed patients. The groups were comparable. Compared to thrombolysis, angioplasty had a higher success rate (68 and 91\% respectively), resulted in less complications and reduced mortality (11 and 2\% respectively), required less revascularization procedures and shorter hospital stay (17 and 13 days, respectively). During the follow-up of survivors, no differences in events or additional mortality were detected at 1 or 5 years. Conclusions: Primary angioplasty is superior as treatment in terms of achieving success and reducing mortality during hospitalization. Evolution after hospitalization is independent of initial therapy.

(Rev Med Chile 2011; 139: 1396-1402).

Key words: Myocardial Infarction; Thrombolytic therapy.
L a enfermedad coronaria es la principal causa de muerte en nuestro país y el infarto agudo ST (IAMST) es su manifestación aguda más letal, para el cual se ha logrado disminuir la mortalidad intrahospitalaria mediante el uso de la terapia de reperfusión (TR $)^{1,2}$. Su utilización se ha consolidado en el mundo y su uso alcanza aproximadamente $67 \%$ en Chile, según la última información del registro $\mathrm{GEMI}^{3}$. Existen dos modalidades básicas de TR, una, la trombolisis endovenosa (TEV), para la cual existen diferentes fármacos en uso, dentro de ellos los más utilizados son la estreptoquinasa (SK) y el activador tisular del plasminógeno (TPA), en Chile, la SK es la más utilizada y es el fármaco aprobado en las normas de garantías explícitas en salud $\mathrm{GES}^{4}$. La segunda opción disponible es la angioplastía coronaria percutánea primaria (inmediata) (APP), inicialmente realizada sólo con balón y en los últimos años, con la aplicación de mallas autoexpandibles o stents intracoronarios. Durante muchos años se discutió en el mundo cual era la mejor alternativa terapéutica y actualmente se reconoce a la APP como la mejor opción ${ }^{5}$ y así lo 
recogen las últimas guías publicadas en el mundo para el manejo del IAM ${ }^{6,7}$, así como la guía chilena publicada por la Sociedad Chilena de Cardiología en el año $2009^{8}$ y la última guía GES para IAMST ${ }^{4}$ en las cuales la TEV se reserva para los casos en que no existe disponibilidad oportuna de APP. En Chile no existen estudios locales adecuados que comparen estas dos alternativas terapéuticas, lo que motivó la realización de este trabajo. El objetivo de este trabajo fue comparar los resultados en pacientes chilenos de la terapia de reperfusión en el IAMST con estreptoquinasa endovenosa versus angioplastía primaria tanto en la evolución intrahospitalaria como en el seguimiento a uno y cinco años plazo.

\section{Pacientes y Método}

Se realizó un estudio de cohorte histórica a partir de un registro prospectivo de todos los pacientes ingresados con IAM a la unidad coronaria de nuestro centro. Se seleccionaron todos los casos con IAMST sometidos a terapia con APP hasta 12 horas de evolución del cuadro según inicio de dolor. Los pacientes seleccionados para angioplastía dependían de la disponibilidad de laboratorio de hemodinamia; sin disponibilidad, se optaba por trombolisis. Se constituyó un grupo de pacientes sometidos a TEV con SK, pareados por sexo y edad ( \pm 3 años) ingresados dentro de 3 meses respecto a cada paciente del grupo APP. Se excluyeron los pacientes ingresados con IAM en shock cardiogénico (Killip IV), condición reconocida como entidad aparte en los estudios. Se compararon los grupos en cuanto a características clínicas de base, factores de riesgo, tratamiento y evolución intrahospitalaria. Se define éxito de la APP a la obtención de arteria abierta, con lesión residual menor o igual a $30 \%$ y flujo timi 2 ó 3 , para la TEV el éxito es definido con la presencia de al menos dos criterios clásicos de reperfusión presentes 9 . Se realizó el seguimiento durante 1 año para mortalidad y otros eventos mayores (reinfarto, accidentes cerebrovascular, isquemia recurrente, necesidad de revascularización, hospitalización por causa cardiovascular) y seguimiento de sobrevida por 5 años. En caso de pacientes cuyos datos no se pudieran obtener de registros hospitalarios se realizó contacto telefónico o visita domiciliaria. Ante falta de datos con estos métodos, para morta- lidad se realizó consulta en registro civil. Se realizó el análisis estadístico en STATA 9.1. Se utilizó la prueba t de Student para variables continuas con distribución normal, prueba no paramétrica de Mann-Withney para variables ordinales y continuas de distribución asimétrica y se utilizó prueba de $\chi^{2}$ para pruebas de proporciones. Se definió un $\mathrm{p}<0,05$ como significativo. Se expresan los resultados como promedios con desviación estándar, o medianas con rango intercuartílico o proporción en $\%$ con intervalo de confianza de $95 \%$ según el tipo de variable. Se calculó el riesgo relativo y su intervalo de confianza de $95 \%$ para desenlaces clínicos con diferencias significativas.

\section{Resultados}

El estudio se inició el 31-03-1993, día en que se efectuó en nuestro centro la primera APP y finalizó el 21-08-2001 con el último paciente sometido a trombolisis, pues desde esa fecha sólo se efectúan APP en IAMST en nuestro centro. Noventa y ocho pacientes forman el grupo APP, 98 el de TEV, de un total de 272 pacientes sometidos a TEV en ese período.

Las características de los grupos se presentan en la Tabla 1. Se observó una edad media de 62 años, predominio de sexo masculino, hipertensión arterial y tabaquismo como factores de riesgo principales, antecedentes previos de probable enfermedad coronaria, aproximadamente en la mitad de los pacientes. No se encontraron diferencias significativas en las características basales de los dos grupos.

En la Tabla 2 se muestran las características clínicas al ingreso, localización del IAM, resultado del tratamiento aplicado y la terapia farmacológica utilizada en los pacientes. Destaca un tiempo promedio desde el inicio del dolor hasta la terapia de 4,5 horas, algo menor para el grupo de TEV, sin alcanzar significación, tampoco hubo diferencias entre ambos grupos en cuanto a presión arterial, frecuencia cardiaca y Killip de ingreso. Existen diferencias en cuanto a la presencia de IAM anterior, que fue más frecuente en el grupo de APP. En relación a resultado del tratamiento, existen diferencias a favor de la APP, con $91 \%$ de éxito versus $68 \%$ de la TEV. En cuanto a la terapia médica asociada se observó un alto porcentaje de uso de aspirina, nitroglicerina endovenosa (ev); heparina ev y beta-bloqueo, sólo se observaron 
ARTÍCULOS DE INVESTIGACIÓN

Comparación entre trombolisis y angioplastía en el tratamiento inicial del infarto agudo al miocardio - H. Ugalde et al

Tabla 1. Características demográficas y antecedentes

\begin{tabular}{|lccc|}
\hline & $\begin{array}{c}\text { APP } \\
\mathbf{n = 9 8}\end{array}$ & $\begin{array}{c}\text { Trombolisis } \\
\mathbf{n}=\mathbf{9 8}\end{array}$ & $\mathbf{p}$ \\
\hline Edad (años) & $62,13 \pm 10,67$ & $62,08 \pm 10,36$ & 0,97 \\
\hline Sexo masculino & $84,79 \%$ & $84,79 \%$ & 1,00 \\
\hline Tabaco & $44,89 \%$ & $55,1 \%$ & 0,15 \\
\hline Hipertensión & $51,02 \%$ & $43,88 \%$ & 0,32 \\
\hline Diabetes mellitus & $19,38 \%$ & $19,38 \%$ & 1,00 \\
\hline Dislipidemia & $17,34 \%$ & $18,36 \%$ & 0,85 \\
\hline Angor previo & $37,75 \%$ & $40,82 \%$ & 0,66 \\
\hline IAM previo & $11,22 \%$ & $8,16 \%$ & 0,47 \\
\hline Cirugía coronaria previa & $1,02 \%$ & $3,06 \%$ & 0,31 \\
\hline Angioplastía previa & $3,06 \%$ & $2,04 \%$ & 0,65 \\
\hline
\end{tabular}

APP $=$ Angioplastía primaria .

Tabla 2. Descripción del evento agudo y tratamiento inicial

\begin{tabular}{|lccc|}
\hline & \multicolumn{1}{|c}{$\begin{array}{c}\text { APP } \\
\mathbf{n = 9 8}\end{array}$} & $\begin{array}{c}\text { Trombolisis } \\
\mathbf{n}=\mathbf{9 8}\end{array}$ & $\mathbf{p}$ \\
\hline Tiempo dolor (hrs) & 4,85 & 4,04 & $0,02^{*}$ \\
\hline $\begin{array}{l}\text { Frecuencia cardiaca } \\
\text { (LPM) }\end{array}$ & $80,74 \pm 15,69$ & $78,01 \pm 17,08$ & 0,24 \\
\hline $\begin{array}{l}\text { Presión arterial } \\
\text { sistólica (mmHg) }\end{array}$ & $131,38 \pm 21,74$ & $132,04 \pm 20,51$ & 0,83 \\
\hline Killip 1 de ingreso & $82,7 \%$ & $80,61 \%$ & 0,78 \\
\hline $\begin{array}{l}\text { Infarto de pared } \\
\text { anterior }\end{array}$ & $61,22 \%$ & $44,9 \%$ & $0,02^{*}$ \\
\hline Aspirina & $98,98 \%$ & $97,96 \%$ & 0,56 \\
\hline$\beta$-Bloqueo & $44,9 \%$ & $48,98 \%$ & 0,57 \\
\hline Nitroglicerina & $94,9 \%$ & $86,73 \%$ & $0,04^{*}$ \\
\hline Heparina & $78,57 \%$ & $46,94 \%$ & $0,00^{*}$ \\
\hline Inhibidores de ECA & $33,67 \%$ & $20,41 \%$ & $0,03^{*}$ \\
\hline Inótropos & $5,1 \%$ & $8,2 \%$ & 0,39 \\
\hline
\end{tabular}

$\mathrm{APP}=$ Angioplastía Primaria. $\mathrm{LPM}=$ Latidos por minuto. $\mathrm{ECA}=$ Enzima convertidora de angiotensina. ${ }^{*}=p$ significativo $<0,05$. diferencias en el uso de heparina, que fue mayor en el grupo de APP.

Todos los pacientes del grupo APP fueron estudiados con angiografía previa a la angioplastía, en el grupo de TEV, $84 \%$ de los pacientes fueron a angiografía coronaria reflejando este número una estrategia del departamento.

En cuanto a complicaciones y eventos intrahospitalarios (Tabla 3), en el grupo de APP se observaron significativamente menos complicaciones generales, menos hipotensión e isquemia recurrente. En relación a eventos mayores, destacó la menor necesidad de revascularización, el menor número de días de hospitalización y la menor mortalidad, todas diferencias significativas a favor del grupo que recibió APP. No se observaron diferencias en el tratamiento recibido al alta entre ambos grupos.

$\mathrm{Al}$ analizar el seguimiento al año y a 5 años (Tabla 4), se logró seguir a más de 95\% de los pacientes en cada grupo. A 1 año de seguimiento no se observaron diferencias significativas en cuanto a eventos isquémicos y necesidad de nueva revascularización, ni en cuanto a mortalidad. A los 5 años, la mortalidad en ambos grupos fue en promedio de $12 \%$, sin diferencias significativas.

Se comparó el grupo total de pacientes que recibieron TEV con el grupo de 98 pacientes seleccionados con TEV para ser comparado con el grupo APP y no se encontraron diferencias entre ambos en ninguno de los puntos analizados.

\section{Discusión}

El IAM ocurre por la oclusión trombótica aguda en una placa ateroesclerótica rota, hecho descrito hace varias décadas ${ }^{10}$ y demostrado sin lugar a dudas en el año 1980 por De Wood et al ${ }^{11}$, quienes confirman la importancia de la oclusión trombótica 
ARTÍCULOS DE INVESTIGACIÓN Comparación entre trombolisis y angioplastía en el tratamiento inicial del infarto agudo al miocardio - H. Ugalde et al

Tabla 3. Evolución hospitalaria

\begin{tabular}{|c|c|c|c|c|}
\hline & $\begin{array}{c}\text { APP } \\
n=98\end{array}$ & $\begin{array}{c}\text { Trombolisis } \\
n=98\end{array}$ & $\mathbf{p}$ & RR [IC 95\%] \\
\hline Necesidad de revascularización: & $9,18 \%$ & $44,9 \%$ & $0,000^{*}$ & $0,2 \quad[0,11-0,40]$ \\
\hline - Angioplastía & $3,06 \%$ & $36,73 \%$ & $0,000 *$ & \\
\hline - Cirugía & $6,12 \%$ & $9,18 \%$ & 0,42 & \\
\hline AVE & $0 \%$ & $3,06 \%$ & 0,08 & \\
\hline Necesidad de trasfusión & $2,04 \%$ & $2,04 \%$ & 1,00 & \\
\hline Insuficiencia cardiaca & $19,39 \%$ & $28,57 \%$ & 0,10 & \\
\hline Hipotensión & $2,04 \%$ & $15,31 \%$ & $0,01 *$ & $0,1 \quad[0,03-0,57]$ \\
\hline Pericarditis & $2,04 \%$ & $3,06 \%$ & 0,65 & \\
\hline Arritmias ventriculares & $9,20 \%$ & $5,10 \%$ & 0,26 & \\
\hline Arritmias supraventriculares & $4,10 \%$ & $3,10 \%$ & 0,70 & \\
\hline BAV & $7,14 \%$ & $2,04 \%$ & 0,08 & \\
\hline Isquemia recurrente [IsqR] & $2,04 \%$ & $14,29 \%$ & $0,001^{*}$ & $0,14[0.03-0,61]$ \\
\hline Reinfarto & $2,04 \%$ & $2,04 \%$ & 1,00 & \\
\hline Mortalidad hospitalaria [MH] & $2,04 \%$ & $11,22 \%$ & $0,009^{*}$ & $0,18[0,04-0,79]$ \\
\hline $\mathrm{MH}$ o reinfarto & $4,08 \%$ & $13,27 \%$ & $0,02^{*}$ & $0,3 \quad[0,10-0,91]$ \\
\hline $\mathrm{MH}$ o reinfarto o AVE & $4,08 \%$ & $16,33 \%$ & $0,004^{*}$ & $0,25[0,09-0,72]$ \\
\hline $\mathrm{MH}$ o reinfarto o AVE o Isq $\mathrm{R}$ & $5,10 \%$ & $26,53 \%$ & $0,000^{*}$ & $0,19[0,08-0,5]$ \\
\hline Días hospitalización promedio & 12,59 & 15,3 & $0,02 *$ & \\
\hline
\end{tabular}

$\mathrm{APP}=$ Angioplastía primaria. $\mathrm{AVE}=$ Accidente vascular encefálico. $\mathrm{BAV}=$ Bloqueo auriculoventricular. ${ }^{*} p$ significativo $<0,05$.

Tabla 4. Eventos y mortalidad al seguimiento

\begin{tabular}{|c|c|c|c|}
\hline & APP & Trombolisis & $\mathbf{p}$ \\
\hline Sobrevivientes & $\mathrm{n}=96$ & $\mathrm{n}=87$ & \\
\hline Pacientes seguidos & $98,96 \%$ & $96,6 \%$ & 0,27 \\
\hline $\begin{array}{l}\text { Eventos totales } \\
\text { primer año }\end{array}$ & $18,95 \%$ & $21,43 \%$ & 0,68 \\
\hline $\begin{array}{l}\text { Mortalidad primer } \\
\text { año }\end{array}$ & $3,13 \%$ & $4,6 \%$ & 0,60 \\
\hline Mortalidad 5 años & $11,45 \%$ & $12,64 \%$ & 0,80 \\
\hline
\end{tabular}

en los estados iniciales del IAMST, estos hechos condujeron a la idea de que lograr eliminar este trombo podría llevar a mejorar el pronóstico y la evolución de un paciente con IAMST. Los estudios iniciales fueron realizados con la aplicación de un agente trombolítico por vía intracoronaria ${ }^{12,13}$ y posteriormente por vía endovenosa, vía más sencilla y rápida, que alcanzó su validación plena con dos grandes estudios en los años 1986-1988, GISSI- $1^{14}$ e ISIS- $2^{15}$, los que demostraron en forma incuestionable la disminución de mortalidad que ocurre con TEV asociada con aspirina. En forma paralela aparece la segunda opción de terapéutica de reperfusión, la $\mathrm{APP}^{16}$. Posteriormente, aparecen los primeros trabajos comparativos entre ambos tratamientos, algunos mostrando a la APP como mejor alternativa de reperfusión ${ }^{17-19}$, otros que no encontraron diferencias ${ }^{20-22}$. Al analizar un metaanálisis publicado en 1997 se observó un mejor resultado a favor de la $\mathrm{APP}^{23}$, esto no se tradujo en un cambio en las guías clínicas del IAMST, las cuales mantuvieron a la TEV como la primera opción de reperfusión ${ }^{24}$. En el año 2003 se publica un nuevo meta-análisis ${ }^{5}$ que demuestra sin lugar a dudas la superioridad de la APP, y estos resultados logran modificar las nuevas guías de manejo del IAM, que consideran a la APP como el tratamiento ideal de reperfusión, cuando es posible ${ }^{6-9}$. 
En nuestro país poco se ha publicado respecto a los resultados de la APP en nuestra población, existen algunas publicaciones referentes a APP, que muestran buenos resultados en forma global con este tratamiento ${ }^{25,26}$, igualmente algunos resultados de terapia trombolítica publicados por el grupo $\mathrm{GEMI}^{27}$, no existiendo un trabajo comparativo adecuado entre ambas terapias. Hay un estudio publicado que comparó ambas alternativas terapéuticas entre dos grupos de pacientes atendidos en distintos centros, uno de ellos, de carácter universitario que efectuaba únicamente APP y otros dos centros de la red asistencial pública que usaba sólo TEV; en este estudio a pesar de existir diferencias demográficas y recibir diferentes terapias farmacológicas entre los grupos, se concluye que la APP es la mejor alternativa de reperfusión ${ }^{28}$. Una reciente publicación compara dos registros chilenos utilizando los pacientes con TEV del registro GEMI y un subgrupo de APP extraído del RENAC (registro de angioplastía en Chile), este estudio muestra resultados similares ${ }^{29}$. Estos dos estudios se basan en grupos diferentes de pacientes, son obtenidos de bases de datos previas y no se diseñaron para el objetivo que pretenden probar.

Nuestro estudio fue posible porque en nuestro centro coexistió la realización de APP o TEV en pacientes que ingresaban con IAMST hasta el año 2001, pues hasta esa fecha, igual como ocurría en el mundo en general, no existía certeza como para considerar a una opción prioritaria sobre la otra, aunque se pensaba que claramente la APP era la mejor opción. En el año 2000 fue presentado en el congreso de cardiología chileno un informe preliminar de estos datos, sustentando la decisión de nuestro centro de definir a la APP como la opción de tratamiento, lo cual se aplica desde el año 2001.

En este trabajo los datos nos muestran dos grupos comparables, adicionalmente el grupo de pacientes seleccionados como TEV es representativo de los que recibían esta terapia, lo que permite realizar y validar los análisis planteados. De esta forma los resultados obtenidos son aplicables a la realidad de nuestro centro, y probablemente a nivel nacional.

Este estudio muestra que la APP es claramente la mejor opción de tratamiento inicial en un IAMST, pues ella determina en el período hospitalario mayor éxito en la reperfusión, menores complicaciones, especialmente en cuanto a hipotensión e isquemia recurrente y menos eventos cardiovasculares, especialmente referido a menos isquemia y menos revascularización, y lo más relevante, menor mortalidad, hallazgos concordantes con lo publicado en la literatura ${ }^{5}$. Es llamativa la baja mortalidad del grupo de APP, pero hay resultados similares publicados por otros grupos ${ }^{30}$, y debe considerarse que no fueron incluidos pacientes en Killip 4. La APP fue inicialmente efectuada sólo con balón y stent provisional (en caso de necesidad, definida por el operador del procedimiento) y en los últimos años del estudio, ante la demostración de que la implantación de stent mejoraba la evolución de los pacientes ${ }^{31-33}$, fue de regla. Este estudio finalizó en el año 2001. Ningún paciente recibió stents liberadores de fármacos, los cuales han mostrado en los últimos estudios lograr mejores resultados, especialmente en relación a la necesidad de nuevas intervenciones ${ }^{34-36}$.

$\mathrm{Al}$ analizar el porcentaje de éxito alcanzado según la terapia aplicada, un factor determinante en los resultados, se observa que con el uso de TEV el resultado es similar a lo mostrado por el grupo $\mathrm{GEMI}^{27}$ y a lo obtenido en diferentes trabajos $^{14,15}$. En cuanto a la APP, nuestros resultados son comparables a los de la literatura internacional ${ }^{5} \mathrm{y}$ ligeramente mejores a los descritos por el grupo $\mathrm{GEMI}^{28}$. La mortalidad hospitalaria fue similar en ambos grupos a lo publicado por GEMI recientemente, así como a la de estudios internacionales ${ }^{5,30}$. En el seguimiento, el cual se logró en forma casi completa ( $98 \%$ en promedio) para los eventos al año y completa para la mortalidad al año y 5 años, se observa que no hay diferencias en la evolución de ambos grupos a largo plazo, en ninguna de las variables analizadas, es decir, se mantiene la ventaja de la APP en cuanto a mortalidad hospitalaria, sin diferencias en cuanto a eventos y mortalidad en el largo plazo.

En síntesis, este estudio confirma en nuestro país lo recogido por las últimas guías publicadas para el manejo del IAM, que indican que en caso de disponer de APP esta debe ser el tratamiento de reperfusión inicial indicado, y dados los datos disponibles en nuestro país ${ }^{29}$, que muestran que en los diferentes grupos que realizan este procedimiento los resultados son similares a los obtenidos por nuestro grupo creemos que debe implementarse esta alternativa terapéutica como opción inicial de manejo del IAMST donde esté disponible, y de ser posible las 24 horas del día, 
los 7 días de la semana, como lo sugieren las guías, para lo cual el gobierno y los sistemas de salud, tanto públicos como privados, deberían considerar su implementación y continuidad. Así también es importante destacar que, en caso de no disponer de esta alternativa, en nuestro país está disponible para todos los pacientes la trombolisis endovenosa, método ampliamente validado en el mundo y, sin duda, efectivo. Se debe recordar que la consulta oportuna del paciente y el diagnóstico a tiempo permiten iniciar las terapias disponibles, por lo que tanto la sospecha diagnóstica como la educación a la comunidad deben ser consideradas como elementos de importancia.

\section{Referencias}

1. Solimano G, Mazzei M. ¿De qué mueren los chilenos hoy?: perspectivas para el largo plazo. Rev Med Chile 2007; 135: 932-938.

2. Departamento de Estadísticas e Información en Salud. Ministerio de Salud de Chile 2007; URL: http//deis. minsal.cl/index.asp.

3. Nazzal C, Campos P, Corvalán R, Lanas F, Bartolucci J, Sanhueza P, et al, en representación del Grupo GEMI, Departamento de Estudios Multicéntricos, Sociedad Chilena de Cardiología y Cirugía Cardiovascular. Impacto del plan AUGE en el tratamiento de pacientes con infarto agudo al miocardio con supradesnivel ST, en hospitales chilenos. Rev Med Chile 2008; 136: 1231-9.

4. http://www.minsal.cl/ici/guiasclinicas/infartoagudo.pdf

5. Keeley E, Boura J, Grines C. Primary angioplasty versus intravenous thrombolytic therapy for acute myocardial infarction: a quantitative review of 23 randomized trials. Lancet 2003; 361: 13-20.

6. Guías de Práctica Clínica de la Sociedad Europea de Cardiología (ESC). Manejo del infarto agudo de miocardio en pacientes con elevación persistente del segmento ST Grupo de Trabajo de la Sociedad Europea de Cardiología (ESC) sobre el manejo del infarto agudo de miocardio con elevación del segmento ST (IAMCEST). Rev Esp Cardiol 2009; 62 (3): e1-e47.

7. 2009 Focused Updates: ACC/AHA Guidelines for the Management of Patients With ST-Elevation Myocardial Infarction (Updating the 2004 Guideline and 2007 Focused Update) and ACC/AHA/SCAI Guidelines on Percutaneous Coronary Intervention (Updating the 2005 Guideline and 2007 Focused Update): A Report of the American College of Cardiology Foundation/American Heart Association Task Force on Practice Guidelines Circulación 2009; 120: 2271-306.
8. Guarda E, Prieto J, Sanhueza P, Dauvergne C, Asenjo R, Corvalán R. Guías 2009 de la Sociedad Chilena de Cardiología para el tratamiento del Infarto Agudo del Miocardio con supradesnivel del ST. Rev Chil Cardiol 2009; 28: 223-54.

9. ACC/AHA guidelines for the management of patients with ST-elevation myocardial infarction: a report of the American College of Cardiology/American Heart Association Task Force on Practice Guidelines (Committee to Revise the 1999 Guidelines for the Management of Patients with Acute Myocardial Infarction). Circulation 2004; 110: e82.

10. Davies M, Woolf N, Robertson W. Pathology of acute myocardial infarction with particular reference to occlusive coronary thrombi. Br Heart J 1976; 38: 659-64.

11. Dewood M, Spores J, Notske R, Mouser L, Burroughs R, Golden M, et al. Prevalence of total coronary occlusion during the early hours of transmural myocardial Infarction. N Engl J Med 1980; 303: 897-902.

12. Rentrop P, Blanke H, Karsh K, Kaiser H, Kostering H, Leitz K. Selective intracoronary thrombolysis in acute myocardial infarction and unstable angina pectoris. Circulation 1981; 63: 307-17.

13. Ganz W, Ninomiya K, Hashida J, Fishbein M, Buchbinder $\mathrm{N}$, Marcus $\mathrm{H}$, et al. Intracoronary thrombolysis in acute myocardial infarction: Experimental background and clinical experience. Am Heart J 1981; 102: 1145-9.

14. GISSI (Gruppo Italiano Per lo Studio Della Streptokinasi Nell Infarto Miocardico) Effectiveness of intravenous thrombolytic treatment in acute myocardial infarction. Lancet 1986; 1: 397-402

15. ISIS-2 (Second International Study of Infarct Survival) Collaborative Group. Randomized trial of intravenous streptokinase, oral aspirin, both or neither among 17187 cases of suspected acute myocardial infarction: ISIS- 2 . Lancet 1988; 2: 349-360.

16. Hartzler G, Rumerford B, Mcconahay D, Johnson W, Mccallister B, Gura G, et al. Percutaneous transluminal coronary angioplasty with and without thrombolytic therapy for treatment of acute myocardial infarction. Am Heart J 1983; 106: 965-73

17. Zijlstra F, De Boer M, Hoornje J, Reiffers S, Reiber J, Suryapranata $\mathrm{H}$. A comparison of immediate coronary angioplasty with intravenous streptokinase in acute myocardial infarction. N Engl J Med 1993; 328: 680-4.

18. Akhras F, Abu Ousa A, Swann G, Duncan H, ChamsiPasha H, Jabbab H. Primary coronary angioplasty or intravenous thrombolysis for patients with acute myocardial infarction? Acute and late follow-up results in a new cardiac unit. J Am Coll Cardiol 1997; 29 (suppl): A235-6. 
19. García E, Elizaga J, Soriano J. Primary angioplasty versus thrombolysis with IPA in anterior myocardial infarction: results from single trial. J Am Coll Cardiol 1997; 29 (suppl): A389.

20. Ribeiro E, Silva L, Carneiro R, D’oliveira L, Gasquez A, Amino J, et al. Randomized trial of direct coronary angioplasty versus intravenous streptokinase in acute myocardial infarction. J Am Coll Cardiol 1993; 22: 37680.

21. Le May M, Labinaz M, Davies R, Marquis J, Laramee L, O’Brien E, et al. Stenting versus thrombolysis in acute myocardial infarction trial (STAT). J Am Coll Cardiol 2001; 37: 985-91.

22. Gibbons R, Holmes D, Reeder G, Guy S, Bailey K, Hopfenspirger $\mathrm{M}$, et al. Immediate angioplasty compared with the administration of a thrombolytic agent followed by conservative treatment for myocardial infarction. N Engl J Med 1993; 328: 685-91.

23. Weaver W, Simes R, Betriu A, Grines C, Zijlstra F, García $\mathrm{E}$, et al. Comparison of primary coronary angioplasty and intravenous thrombolytic therapy for acute myocardial infarction: a quantitative review. JAMA 1997; 278: 2093-8.

24. 1999 update: ACC/AHA guidelines for the management of patients with acute myocardial infarction. A report of the American College of Cardiology/American Heart Association Task Force on Practice Guidelines (Committee on Management of Acute Myocardial Infarction). J Am Coll Cardiol 1999; 34 (3): 890-911.

25. Chamorro H, Ducci H, Mathei R, Alcaino M, Florenzano F, Ramírez A, et al. Angioplastía coronaria primaria como tratamiento de elección en las primeras 6 horas del infarto de miocardio. Rev Med Chile 1995; 123: 72734 .

26. Ugalde H, Ramírez A, Benavente D, Antúnez M, García S, Dussaillant G, et al. Angioplastía en Infarto Agudo al Miocardio. Resultado inicial y seguimiento alejado. Rev Med Chile 1999; 127: 565-75.

27. Prieto J, Sanhueza C, Martínez N, Nazzal C, Corvalán R, Castro P, et al, del Grupo de Estudio Multicéntrico del Infarto (GEMI). Mortalidad intrahospitalaria en hombres y mujeres según terapias de reperfusión en infarto agudo del miocardio con supradesnivel del ST del Grupo de Estudio Multicéntrico del Infarto (GEMI). Rev Med Chile 2008; 136: 143-50.
28. Greig D, Corbalán R, Castro P, Campos P, Lamich R, Yovaniniz $\mathrm{P}$, et al. Impacto de la trombolisis y de la angioplastia primaria en pacientes con infarto agudo del miocardio tratados en centros hospitalarios terciarios. Rev Med Chile 2008; 136: 1098-106.

29. Martínez A, Nazzal C, Fajuri A, Barra L, Mayerson A, Cavada G, et al. Mortalidad por infarto al miocardio en Chile: comparación de los registros de angioplastía primaria versus trombolisis. Rev Chil Cardiol 2010; 29: 29-36.

30. Chen K, Rha S, Li Y, Poddar K, Jin Z, Minami Y, et al. Triple versus Dual Antiplatelet Therapy in Patients With Acute ST-Segment Elevation Myocardial Infarction Undergoing Primary Percutaneous Coronary Intervention. Circulation 2009; 119: 3207-14.

31. Grines C, Cox D, Stone G, García E, Mattos L, Giambartolomei A, et al. Coronary angioplasty with or without stent implantation for acute myocardial infarction. Stent Primary Angioplasty in Myocardial Infarction Study Group. N Engl J Med 1999; 341: 1949-56.

32. Maillard L, Hamon M, Khalife K, Steg P, Beygui F, Guermonprez J, et al. A comparison of systematic stenting and conventional balloon angioplasty during primary percutaneous transluminal coronary angioplasty for acute myocardial acute myocardial infarction. STENTIM-2 Investigators. J Am Coll Cardiol 2000; 35: 172936.

33. Zhu M, Feit A, Chadow H, Alam M, Kwan T, Clarck L. Primary stent implantation compared with primary balloon angioplasty for acute myocardial infarction: a meta analysis of randomised clinical trials. Am J Cardiol 2001; 88: 297-301.

34. Mauri L, Silbaugh T, Garg P, Wolf R, Zelevinsky K, Lovett A, et al. Drug-Eluting or Bare-Metal Stents for Acute Myocardial Infarction. N Engl J Med 2008; 359: 1330-42.

35. Stone G, Lansky A, Pocock S, Gersh B, Dangas G, Wong $\mathrm{CH}$, et al, for the HORIZONS-AMI Trial Investigators*. Paclitaxel-Eluting Stents versus Bare-Metal Stents in Acute Myocardial Infarction.N Engl J Med 2009; 360: 1946-59.

36. Brar SS, León M, Stone G, Mehran R, Moses J, Brar SK, et al. Use of Drug-Eluting Stents in Acute Myocardial Infarction A Systematic Review and Meta-Analysis. J Am Coll Cardiol 2009; 53: 1677-89. 